\title{
Approximation of solutions to an equilibrium problem in a nonuniformly smooth Banach space
}

Juan Zhao*

\section{"Correspondence:}

zhaojuanyu@126.com

School of Mathematics and

Information Science, North China

University of Water Resources and

Electric Power, Zhengzhou, China

\begin{abstract}
An equilibrium problem based on a projection algorithm is investigated. A strong convergence theorem for solutions of the equilibrium problems is established in a nonuniformly smooth Banach space.
\end{abstract}

Keywords: equilibrium problem; fixed point; quasi- $\phi$-nonexpansive mapping; projection

\section{Introduction}

Recently, equilibrium problems have been studied as an effective and powerful tool for studying a wide class of real world problems which arise in economics, finance, image reconstruction, ecology, transportation, and networks; see [1-16] and the references therein. It is well known that equilibrium problems include many important problems in nonlinear analysis and optimization such as the Nash equilibrium problem, variational inequalities, complementarity problems, vector optimization problems, fixed point problems, saddle point problems, and game theory. For the solutions of equilibrium problems, there are several algorithms to solve the problem; see [17-28] and the references therein. However, most of these results are obtained in the framework of Hilbert spaces or uniformly smooth Banach spaces.

The purpose of this paper is to study solution problems of an equilibrium problem based on a projection algorithm in a nonuniformly smooth Banach space. The organization of this paper is as follows. In Section 2, we provide some necessary preliminaries. In Section 3, a projection algorithm is introduced and the convergence analysis is given. A strong convergence theorem is established in a nonuniformly smooth Banach space. Applications of the main results are also discussed in this section.

\section{Preliminaries}

Let $E$ be a real Banach space, and let $E^{*}$ be the dual space of $E$. We denote by $J$ the normalized duality mapping from $E$ to $2^{E^{*}}$ defined by

$$
J x=\left\{f^{*} \in E^{*}:\left\langle x, f^{*}\right\rangle=\|x\|^{2}=\left\|f^{*}\right\|^{2}\right\},
$$

where $\langle\cdot, \cdot\rangle$ denotes the generalized duality pairing. A Banach space $E$ is said to be strictly convex if $\left\|\frac{x+y}{2}\right\|<1$ for all $x, y \in E$ with $\|x\|=\|y\|=1$ and $x \neq y$. It is said to be uniformly

(c) 2013 Zhao: licensee Springer. This is an Open Access article distributed under the terms of the Creative Commons Attribution License (http://creativecommons.org/licenses/by/2.0), which permits unrestricted use, distribution, and reproduction in any medium, provided the original work is properly cited. 
convex if $\lim _{n \rightarrow \infty}\left\|x_{n}-y_{n}\right\|=0$ for any two sequences $\left\{x_{n}\right\}$ and $\left\{y_{n}\right\}$ in $E$ such that $\left\|x_{n}\right\|=$ $\left\|y_{n}\right\|=1$ and $\lim _{n \rightarrow \infty}\left\|\frac{x_{n}+y_{n}}{2}\right\|=1$. Let $U_{E}=\{x \in E:\|x\|=1\}$ be the unit sphere of $E$. Then the Banach space $E$ is said to be smooth provided

$$
\lim _{t \rightarrow 0} \frac{\|x+t y\|-\|x\|}{t}
$$

exists for each $x, y \in U_{E}$. It is also said to be uniformly smooth if the above limit is attained uniformly for $x, y \in U_{E}$. It is well known that if $E$ is uniformly smooth, then $J$ is uniformly norm-to-norm continuous on each bounded subset of $E$. It is also well known that $E$ is uniformly smooth if and only if $E^{*}$ is uniformly convex.

Recall that a Banach space $E$ enjoys the Kadec-Klee property if for any sequence $\left\{x_{n}\right\} \subset E$ and $x \in E$ with $x_{n} \rightarrow x$ and $\left\|x_{n}\right\| \rightarrow\|x\|$, then $\left\|x_{n}-x\right\| \rightarrow 0$ as $n \rightarrow \infty$. For more details on the Kadec-Klee property, the readers can refer to [29] and the references therein. It is well known that if $E$ is a uniformly convex Banach space, then $E$ enjoys the Kadec-Klee property. In this paper, we use $\rightarrow$ and $\rightarrow$ to denote the strong convergence and weak convergence, respectively.

Let $C$ be a nonempty subset of $E$. Recall that a mapping $Q: C \rightarrow E^{*}$ is said to be monotone iff

$$
\langle x-y, Q x-Q y\rangle \geq 0, \quad \forall x, y \in C .
$$

$Q: C \rightarrow E^{*}$ is said to be $\alpha$-inverse-strongly monotone iff there exists a positive real number $\alpha$ such that

$$
\langle x-y, Q x-Q y\rangle \geq \alpha\|Q x-Q y\|^{2}, \quad \forall x, y \in C .
$$

Recall also that a monotone mapping $Q$ is said to be maximal iff its graph $\operatorname{Graph}(Q)=$ $\{(x, f): f \in Q x\}$ is not properly contained in the graph of any other monotone mapping. It is known that a monotone mapping $Q$ is maximal iff for $(x, f) \in E \times E^{*},\langle x-y, f-g\rangle \geq 0$ for every $(y, g) \in \operatorname{Graph}(Q)$ implies $f \in Q x$. An operator $Q$ from $C$ into $E$ is said to be hemicontinuous if for all $x, y \in C$, the mapping $f$ of $[0,1]$ into $E$ defined by $f(t)=Q(t x+(1-t) y)$ is continuous with respect to the weak* topology of $E^{*}$. Let $f$ be a bifunction from $C \times C$ to $\mathbb{R}$, where $\mathbb{R}$ denotes the set of real numbers. In this paper, we investigate the following equilibrium problem. Find $p \in C$ such that

$$
f(p, y) \geq 0, \quad \forall y \in C
$$

We use $E P(f)$ to denote the solution set of equilibrium problem (2.1). That is,

$$
E P(f)=\{p \in C: f(p, y) \geq 0, \forall y \in C\} .
$$

Given a mapping $Q: C \rightarrow E^{*}$, let

$$
f(x, y)=\langle Q x, y-x\rangle, \quad \forall x, y \in C .
$$


Then $p \in E P(f)$ iff $p$ is a solution of the following variational inequality. Find $p$ such that

$$
\langle Q p, y-p\rangle \geq 0, \quad \forall y \in C
$$

In order to study the solution problem of equilibrium problem (2.1), we assume that $f$ satisfies the following conditions:

(A1) $f(x, x)=0, \forall x \in C$;

(A2) $f$ is monotone, i.e., $f(x, y)+f(y, x) \leq 0, \forall x, y \in C$;

(A3)

$$
\limsup _{t \downarrow 0} f(t z+(1-t) x, y) \leq f(x, y), \quad \forall x, y, z \in C ;
$$

(A4) for each $x \in C, y \mapsto f(x, y)$ is convex and weakly lower semi-continuous.

As we all know, if $C$ is a nonempty closed convex subset of a Hilbert space $H$ and $P_{C}: H \rightarrow C$ is the metric projection of $H$ onto $C$, then $P_{C}$ is nonexpansive. This fact actually characterizes Hilbert spaces and consequently it is not available in more general Banach spaces. In this connection, Alber [30] recently introduced a generalized projection operator $\Pi_{C}$ in a Banach space $E$ which is an analogue of the metric projection $P_{C}$ in Hilbert spaces.

Next, we assume that $E$ is a smooth Banach space. Consider the functional defined by

$$
\phi(x, y)=\|x\|^{2}-2\langle x, J y\rangle+\|y\|^{2}, \quad \forall x, y \in E .
$$

Observe that in a Hilbert space $H$, the equality is reduced to $\phi(x, y)=\|x-y\|^{2}, x, y \in H$. The generalized projection $\Pi_{C}: E \rightarrow C$ is a map that assigns to an arbitrary point $x \in E$ the minimum point of the functional $\phi(x, y)$, that is, $\Pi_{C} x=\bar{x}$, where $\bar{x}$ is the solution to the minimization problem

$$
\phi(\bar{x}, x)=\min _{y \in C} \phi(y, x)
$$

The existence and uniqueness of the operator $\Pi_{C}$ follow from the properties of the functional $\phi(x, y)$ and strict monotonicity of the mapping $J$; see, for example, [29] and [30]. In Hilbert spaces, $\Pi_{C}=P_{C}$. It is obvious from the definition of a function $\phi$ that

$$
(\|x\|-\|y\|)^{2} \leq \phi(x, y) \leq(\|y\|+\|x\|)^{2}, \quad \forall x, y \in E,
$$

and

$$
\phi(x, y)=\phi(x, z)+\phi(z, y)+2\langle x-z, J z-J y\rangle, \quad \forall x, y, z \in E .
$$

Let $T: C \rightarrow C$ be a mapping. In this paper, we use $F(T)$ to denote the fixed point set of $T$. A point $p$ in $C$ is said to be an asymptotic fixed point of $T$ iff $C$ contains a sequence $\left\{x_{n}\right\}$ which converges weakly to $p$ such that $\lim _{n \rightarrow \infty}\left\|x_{n}-T x_{n}\right\|=0$. The set of asymptotic fixed points of $T$ will be denoted by $\widetilde{F}(T) . T$ is said to be relatively nonexpansive iff $\widetilde{F}(T)=F(T) \neq \emptyset$ and $\phi(p, T x) \leq \phi(p, x)$ for all $x \in C$ and $p \in F(T)$. $T$ is said to be quasi- $\phi$ nonexpansive iff $F(T) \neq \emptyset$ and $\phi(p, T x) \leq \phi(p, x)$ for all $x \in C$ and $p \in F(T)$. 
Remark 2.1 The class of quasi- $\phi$-nonexpansive mappings is more general than the class of relatively nonexpansive mappings which requires the restriction: $F(T)=\widetilde{F}(T)$.

Remark 2.2 The class of quasi- $\phi$-nonexpansive mappings is a generalization of quasinonexpansive mappings in Hilbert spaces.

In this paper, we investigate the solution problem of equilibrium problem (2.1) based on a projection algorithm. A strong convergence theorem for solutions of the equilibrium problems is established in a reflexive, strictly convex, and smooth Banach space such that both $E$ and $E^{*}$ have the Kadec-Klee property.

In order to give our main results, we need the following lemmas.

Lemma 2.3 [30] Let C be a nonempty, closed, and convex subset of a smooth Banach space $E$, and $x \in E$. Then $x_{0}=\Pi_{C} x$ if and only if

$$
\left\langle x_{0}-y, J x-J x_{0}\right\rangle \geq 0, \quad \forall y \in C
$$

Lemma 2.4 [30] Let E be a reflexive, strictly convex, and smooth Banach space, let $C$ be a nonempty, closed, and convex subset of $E$, and $x \in E$. Then

$$
\phi\left(y, \Pi_{C} x\right)+\phi\left(\Pi_{C} x, x\right) \leq \phi(y, x), \quad \forall y \in C .
$$

Lemma 2.5 [30] Let E be a reflexive, strictly convex, and smooth Banach space. Then

$$
\phi(x, y)=0 \quad \Longleftrightarrow \quad x=y .
$$

The following lemma can be obtained from [15] and [31].

Lemma 2.6 Let $C$ be a closed convex subset of a smooth, strictly convex, and reflexive Banach space E. Let $f$ be a bifunction from $C \times C$ to $\mathbb{R}$ satisfying (A1)-(A4). Let $r>0$ and $x \in E$. Then

(a) There exists $z \in C$ such that

$$
f(z, y)+\frac{1}{r}\langle y-z, J z-J x\rangle \geq 0, \quad \forall y \in C .
$$

(b) Define a mapping $T_{r}: E \rightarrow C$ by

$$
S_{r} x=\left\{z \in C: f(z, y)+\frac{1}{r}\langle y-z, J z-J x\rangle, \forall y \in C\right\} .
$$

Then the following conclusions hold:

(1) $F\left(S_{r}\right)=E P(f)$;

(2) $S_{r}$ is a firmly nonexpansive-type mapping, i.e., for all $x, y \in E$,

$$
\left\langle S_{r} x-S_{r} y, J S_{r} x-J S_{r} y\right\rangle \leq\left\langle S_{r} x-S_{r} y, J x-J y\right\rangle
$$

(3) $S_{r}$ is single-valued; 
(4)

$$
\phi\left(q, S_{r} x\right)+\phi\left(S_{r} x, x\right) \leq \phi(q, x), \quad \forall q \in F\left(S_{r}\right)
$$

(5) $E P(f)$ is closed and convex;

(6) $S_{r}$ is quasi- $\phi$-nonexpansive.

\section{Main results}

Theorem 3.1 Let E be a reflexive, strictly convex, and smooth Banach space such that both $E$ and $E^{*}$ have the Kadec-Klee property. Let $C$ be a nonempty, closed, and convex subset of $E$. Letf be a bifunction from $C \times C$ to $\mathbb{R}$ satisfying (A1)-(A4) such that $E F(f)$ is nonempty. Let $\left\{x_{n}\right\}$ be a sequence generated in the following manner:

$$
\left\{\begin{array}{l}
x_{0} \in E \quad \text { chosen arbitrarily, } \\
C_{1}=C, \\
x_{1}=\Pi_{C_{1}} x_{0}, \\
y_{n} \in C \text { such that } f\left(y_{n}, y\right)+\frac{1}{r_{n}}\left\langle y-y_{n}, J y_{n}-J x_{n}\right\rangle \geq 0, \quad \forall y \in C, \\
C_{n+1}=\left\{z \in C_{n}: \phi\left(z, y_{n}\right) \leq \phi\left(z, x_{n}\right)\right\}, \\
x_{n+1}=\Pi_{C_{n+1}} x_{1},
\end{array}\right.
$$

where $\left\{r_{n}\right\}$ is a real sequence such that $\liminf _{n \rightarrow \infty} r_{n}>0$. Then the sequence $\left\{x_{n}\right\}$ converges strongly to $\Pi_{E P(f)} x_{1}$, where $\Pi_{E P(f)}$ is the generalized projection from $E$ onto $E P(f)$.

Proof First, we show that $C_{n}$ is closed and convex, that the projection on it is well defined. It is obvious that $C_{1}=C$ is closed and convex. Suppose that $C_{m}$ is closed and convex for some $m \in \mathbb{N}$. We next prove that $C_{m+1}$ is also closed and convex for the same $m$. Let For $z_{1}, z_{2} \in C_{m+1}$, we see that $z_{1}, z_{2} \in C_{m}$. It follows that $z=t z_{1}+(1-t) z_{2} \in C_{m}$, where $t \in(0,1)$. Notice that

$$
\phi\left(z_{1}, y_{m}\right) \leq \phi\left(z_{1}, x_{m}\right) \quad \text { and } \quad \phi\left(z_{1}, y_{h}\right) \leq \phi\left(z_{1}, x_{m}\right)
$$

The above inequalities are equivalent to

$$
2\left\langle z_{1}, J x_{m}-J y_{m}\right\rangle \leq\left\|x_{m}\right\|^{2}-\left\|y_{m}\right\|^{2}
$$

and

$$
2\left\langle z_{2}, J x_{m}-J y_{m}\right\rangle \leq\left\|x_{m}\right\|^{2}-\left\|y_{m}\right\|^{2} .
$$

Multiplying $t$ and $(1-t)$ on both sides of (3.1) and (3.2), respectively, yields that

$$
2\left\langle z, J x_{m}-J y_{m}\right\rangle \leq\left\|x_{m}\right\|^{2}-\left\|y_{m}\right\|^{2} .
$$

That is,

$$
\phi\left(z, y_{m}\right) \leq \phi\left(z, x_{m}\right) .
$$


This gives that $C_{m+1}$ is closed and convex. Then $C_{n}$ is closed and convex. Now, we are in a position to prove that $E P(f) \subset C_{n}$. $E P(f) \subset C_{1}=C$ is obvious. Suppose that $E P(f) \subset C_{m}$ for some $m \in \mathbb{N}$. Fix $p \in E P(f) \subset C_{m}$. It follows that

$$
\phi\left(p, y_{m}\right)=\phi\left(p, S_{r_{m}} x_{m}\right) \leq \phi\left(p, x_{m}\right)
$$

which implies that $p \in C_{m+1}$. This proves that $E P(f) \subset C_{n}$. In the light of $x_{n}=\Pi_{C_{n}} x_{1}$, from Lemma 2.3, we find that $\left\langle x_{n}-z, J x_{1}-J x_{n}\right\rangle \geq 0$ for any $z \in C_{n}$. It follows from $E P(f) \subset C_{n}$ that

$$
\left\langle x_{n}-w, J x_{1}-J x_{n}\right\rangle \geq 0, \quad \forall w \in E P(f) .
$$

It follows from Lemma 2.4 that

$$
\begin{aligned}
\phi\left(x_{n}, x_{1}\right) & =\phi\left(\Pi_{C_{n}} x_{1}, x_{1}\right) \\
& \leq \phi\left(\Pi_{F(T)} x_{1}, x_{1}\right)-\phi\left(\Pi_{F(T)} x_{1}, x_{n}\right) \\
& \leq \phi\left(\Pi_{F(T)} x_{1}, x_{1}\right) .
\end{aligned}
$$

This implies that the sequence $\left\{\phi\left(x_{n}, x_{1}\right)\right\}$ is bounded. It follows from (2.3) that the sequence $\left\{x_{n}\right\}$ is also bounded. Since the space is reflexive, we may assume that $x_{n} \rightarrow \bar{x}$. Since $C_{n}$ is closed and convex, we find that $\bar{x} \in C_{n}$. On the other hand, we see from the weak lower semicontinuity of the norm that

$$
\begin{aligned}
\phi\left(\bar{x}, x_{1}\right) & =\|\bar{x}\|^{2}-2\left\langle\bar{x}, J x_{1}\right\rangle+\left\|x_{1}\right\|^{2} \\
& \leq \liminf _{n \rightarrow \infty}\left(\left\|x_{n}\right\|^{2}-2\left\langle x_{n}, J x_{1}\right\rangle+\left\|x_{1}\right\|^{2}\right) \\
& =\liminf _{n \rightarrow \infty} \phi\left(x_{n}, x_{1}\right) \\
& \leq \limsup _{n \rightarrow \infty} \phi\left(x_{n}, x_{1}\right) \\
& \leq \phi\left(\bar{x}, x_{1}\right),
\end{aligned}
$$

which implies that $\lim _{n \rightarrow \infty} \phi\left(x_{n}, x_{1}\right)=\phi\left(\bar{x}, x_{1}\right)$. Hence, we have $\lim _{n \rightarrow \infty}\left\|x_{n}\right\|=\|\bar{x}\|$. In view of the Kadec-Klee property of $E$, we find that $x_{n} \rightarrow \bar{x}$ as $n \rightarrow \infty$. Next, we show that $p \in$ $E F(f)$. In view of construction of $x_{n+1}=\prod_{E P(f)} x_{1} \in C_{n+1} \subset C_{n}$, we find that

$$
\begin{aligned}
\phi\left(x_{n+1}, x_{n}\right) & =\phi\left(x_{n+1}, \Pi_{C_{n}} x_{1}\right) \\
& \leq \phi\left(x_{n+1}, x_{1}\right)-\phi\left(\Pi_{C_{n}} x_{1}, x_{1}\right) \\
& =\phi\left(x_{n+1}, x_{1}\right)-\phi\left(x_{n}, x_{1}\right) .
\end{aligned}
$$

Since $x_{n}=\Pi_{C_{n}} x_{1}$ and $x_{n+1}=\Pi_{C_{n+1}} x_{1} \in C_{n+1} \subset C_{n}$, we arrive at $\phi\left(x_{n}, x_{1}\right) \leq \phi\left(x_{n+1}, x_{1}\right)$. This shows that $\left\{\phi\left(x_{n}, x_{1}\right)\right\}$ is nondecreasing. It follows from the boundedness that $\lim _{n \rightarrow \infty} \phi\left(x, x_{1}\right)$ exists. This implies from (3.4) that

$$
\lim _{n \rightarrow \infty} \phi\left(x_{n+1}, x_{n}\right)=0
$$


Since $x_{n+1}=\Pi_{C_{n+1}} x_{1} \in C_{n+1}$, we arrive at $\phi\left(x_{n+1}, y_{n}\right) \leq \phi\left(x_{n+1}, x_{n}\right)$. It follows that

$$
\lim _{n \rightarrow \infty} \phi\left(x_{n+1}, y_{n}\right)=0
$$

In view of (2.3), we see that $\lim _{n \rightarrow \infty}\left(\left\|x_{n+1}\right\|-\left\|y_{n}\right\|\right)=0$. This in turn implies that $\lim _{n \rightarrow \infty}\left\|y_{n}\right\|=\|\bar{x}\|$. It follows that $\lim _{n \rightarrow \infty}\left\|J y_{n}\right\|=\|J \bar{x}\|$. This implies that $\left\{J y_{n}\right\}$ is bounded. Note that both $E$ and $E^{*}$ are reflexive. We may assume that $J y_{n} \rightarrow y^{*} \in E^{*}$. In view of the reflexivity of $E$, we see that $J(E)=E^{*}$. This shows that there exists an element $y \in E$ such that $J y=y^{*}$. It follows that

$$
\begin{aligned}
\phi\left(x_{n+1}, y_{n}\right) & =\left\|x_{n+1}\right\|^{2}-2\left\langle x_{n+1}, J y_{n}\right\rangle+\left\|y_{n}\right\|^{2} \\
& =\left\|x_{n+1}\right\|^{2}-2\left\langle x_{n+1}, J y_{n}\right\rangle+\left\|J y_{n}\right\|^{2} .
\end{aligned}
$$

Taking liminf $\operatorname{in}_{n \rightarrow \infty}$ on both sides of the equality above yields that

$$
\begin{aligned}
0 & \geq\|\bar{x}\|^{2}-2\left\langle\bar{x}, y^{*}\right\rangle+\left\|y^{*}\right\|^{2} \\
& =\|\bar{x}\|^{2}-2\langle\bar{x}, J y\rangle+\|J y\|^{2} \\
& =\|\bar{x}\|^{2}-2\langle\bar{x}, J y\rangle+\|y\|^{2} \\
& =\phi(\bar{x}, y) .
\end{aligned}
$$

That is, $\bar{x}=y$, which in turn implies that $y^{*}=J \bar{x}$. It follows that $J y_{n} \rightarrow J \bar{x} \in E^{*}$. Since $E^{*}$ enjoys the Kadec-Klee property, we obtain that $\lim _{n \rightarrow \infty} J y_{n}=J \bar{x}$. Notice that

$$
\left\|J x_{n}-J y_{n}\right\| \leq\left\|J x_{n}-J \bar{x}\right\|+\left\|J \bar{x}-J y_{n}\right\|
$$

It follows that $\lim _{n \rightarrow \infty}\left\|J x_{n}-J y_{n}\right\|=0$. From the restriction on the sequence $\left\{r_{n}\right\}$, we find that

$$
\lim _{n \rightarrow \infty} \frac{\left\|J x_{n}-J y_{n}\right\|}{r_{n}}=0 .
$$

In view of $y_{n}=S_{r_{n}} x_{n}$, we see that

$$
f\left(y_{n}, y\right)+\frac{1}{r_{n}}\left\langle y-y_{n}, J y_{n}-J x_{n}\right\rangle \geq 0, \quad \forall y \in C .
$$

It follows from (A2) that

$$
\left\|y-y_{n}\right\| \frac{\left\|J y_{n}-J x_{n}\right\|}{r_{n}} \geq \frac{1}{r_{n}}\left\langle y-y_{n}, J y_{n}-J x_{n}\right\rangle \geq-f\left(y_{n}, y\right) \geq f\left(y, y_{n}\right), \quad \forall y \in C .
$$

By taking the limit as $n \rightarrow \infty$ in the above inequality, from (A4) we obtain that

$$
f(y, \bar{x}) \leq 0, \quad \forall y \in C
$$

For $0<t<1$ and $y \in C$, define $y_{t}=t y+(1-t) \bar{x}$. It follows that $y_{t} \in C$, which yields that $f\left(y_{t}, \bar{x}\right) \leq 0$. It follows from (A1) and (A4) that

$$
0=f\left(y_{t}, y_{t}\right) \leq t f\left(y_{t}, y\right)+(1-t) f\left(y_{t}, \bar{x}\right) \leq t f\left(y_{t}, y\right)
$$


That is,

$$
f\left(y_{t}, y\right) \geq 0
$$

Letting $t \downarrow 0$, we obtain from (A3) that $f(\bar{x}, y) \geq 0, \forall y \in C$. This implies that $\bar{x} \in E P(f)$.

Finally, we prove that $\bar{x}=\Pi_{E P(f)} x_{1}$. Letting $n \rightarrow \infty$ in (3.3), we see that

$$
\left\langle\bar{x}-w, J x_{1}-J \bar{x}\right\rangle \geq 0, \quad \forall w \in E P(f) .
$$

In the light of Lemma 2.3, we find that $\bar{x}=\Pi_{E P(f)} x_{1}$. This completes the proof.

We remark that $L^{p}$, where $p>1$ is a space which satisfies the restriction in Theorem 3.1. Since every uniformly convex and uniformly smooth Banach space is a reflexive, strictly convex, and smooth Banach space such that both $E$ and $E^{*}$ have the Kadec-Klee property, we find from Theorem 3.1 the following result.

Corollary 3.2 Let E be a uniformly convex and uniformly smooth Banach space. Let C be a nonempty, closed, and convex subset of $E$. Let $f$ be a bifunction from $C \times C$ to $\mathbb{R}$ satisfying (A1)-(A4) such that $E F(f)$ is nonempty. Let $\left\{x_{n}\right\}$ be a sequence generated in the following manner:

$$
\left\{\begin{array}{l}
x_{0} \in E \quad \text { chosen arbitrarily, } \\
C_{1}=C, \\
x_{1}=\Pi_{C_{1}} x_{0}, \\
y_{n} \in C \text { such that } f\left(y_{n}, y\right)+\frac{1}{r_{n}}\left\langle y-y_{n}, J y_{n}-J x_{n}\right\rangle \geq 0, \quad \forall y \in C, \\
C_{n+1}=\left\{z \in C_{n}: \phi\left(z, y_{n}\right) \leq \phi\left(z, x_{n}\right)\right\}, \\
x_{n+1}=\Pi_{C_{n+1}} x_{1},
\end{array}\right.
$$

where $\left\{r_{n}\right\}$ is a real sequence such that $\liminf _{n \rightarrow \infty} r_{n}>0$. Then the sequence $\left\{x_{n}\right\}$ converges strongly to $\Pi_{E P(f)} x_{1}$, where $\Pi_{E P(f)}$ is the generalized projection from $E$ onto $E P(f)$.

In the framework of Hilbert spaces, we find from Theorem 3.1 the following result.

Corollary 3.3 Let E be a Hilbert space. Let $C$ be a nonempty, closed, and convex subset of E. Let $f$ be a bifunction from $C \times C$ to $\mathbb{R}$ satisfying (A1)-(A4) such that $E F(f)$ is nonempty. Let $\left\{x_{n}\right\}$ be a sequence generated in the following manner:

$$
\left\{\begin{array}{l}
x_{0} \in E \quad \text { chosen arbitrarily, } \\
C_{1}=C, \\
x_{1}=P_{C_{1}} x_{0}, \\
y_{n} \in C \text { such that } f\left(y_{n}, y\right)+\frac{1}{r_{n}}\left\langle y-y_{n}, y_{n}-x_{n}\right\rangle \geq 0, \quad \forall y \in C, \\
C_{n+1}=\left\{z \in C_{n}:\left\|z-y_{n}\right\|^{2} \leq\left\|z-x_{n}\right\|^{2}\right\}, \\
x_{n+1}=P_{C_{n+1}} x_{1},
\end{array}\right.
$$

where $\left\{r_{n}\right\}$ is a real sequence such that $\liminf _{n \rightarrow \infty} r_{n}>0$. Then the sequence $\left\{x_{n}\right\}$ converges strongly to $P_{E P(f)} x_{1}$, where $P_{E P(f)}$ is the metric projection from $E$ onto $E P(f)$. 
Proof Notice that $\phi(x, y)=\|x-y\|^{2}$. The generalized metric projection is reduced to the metric projection and the normalized duality mapping $J$ is reduced to the identity mapping $I$ in Hilbert spaces. The result can be obtained from Theorem 3.1 immediately.

\section{Competing interests}

The author declares that they have no competing interests.

\section{Acknowledgements}

The author is grateful to the editor and the reviewers for their suggestions which improved the contents of the article.

Received: 16 April 2013 Accepted: 17 June 2013 Published: 19 August 2013

\section{References}

1. Censor, Y, Cohen, N, Kutscher, T, Shamir, J: Summed squared distance error reduction by simultaneous multiprojections and applications. Appl. Math. Comput. 126, 157-179 (2002)

2. liduka, H: Fixed point optimization algorithm and its application to network bandwidth allocation. J. Comput. Appl. Math. 236, 1733-1742 (2012)

3. Qin, X, Chang, SS, Cho, YJ: Iterative methods for generalized equilibrium problems and fixed point problems with applications. Nonlinear Anal. 11, 2963-2972 (2010)

4. Dhage, BC, Jadhav, NS: Differential inequalities and comparison theorems for first order hybrid integro-differential equations. Adv. Inequal. Appl. 2, 61-80 (2013)

5. Abdel-Salam, HS, Al-Khaled, K: Variational iteration method for solving optimization problems. J. Math. Comput. Sci. 2 1475-1497 (2012)

6. Qin, X, Cho, SY, Kang, SM: Strong convergence of shrinking projection methods for quasi- $\boldsymbol{\phi}$-nonexpansive mappings and equilibrium problems. J. Comput. Appl. Math. 234, 750-760 (2010)

7. liduka, H: Strong convergence for an iterative method for the triple-hierarchical constrained optimization problem. Nonlinear Anal. 71, e1292-e1297 (2009)

8. Noor, MA, Noor, Kl, Waseem, M: Decomposition method for solving system of linear equations. Eng. Math. Lett. 2, 34-41 (2013)

9. Cho, SY, Kang, SM: Approximation of fixed points of pseudocontraction semigroups based on a viscosity iterative process. Appl. Math. Lett. 24, 224-228 (2011)

10. Zegeye, $\mathrm{H}$, Shahzad, N: Strong convergence theorem for a common point of solution of variational inequality and fixed point problem. Adv. Fixed Point Theory 2, 374-397 (2012)

11. Qin, X, Cho, SY, Kang, SM: On hybrid projection methods for asymptotically quasi- $\phi$-nonexpansive mappings. Appl. Math. Comput. 215, 3874-3883 (2010)

12. Al-Bayati, AY, Al-Kawaz, RZ: A new hybrid WC-FR conjugate gradient-algorithm with modified secant condition for unconstrained optimization. J. Math. Comput. Sci. 2, 937-966 (2012)

13. Shen, J, Pang, LP: An approximate bundle method for solving variational inequalities. Commun. Optim. Theory 1 , 1-18 (2012)

14. Qin, X, Cho, YJ, Kang, SM: Convergence theorems of common elements for equilibrium problems and fixed point problems in Banach spaces. J. Comput. Appl. Math. 225, 20-30 (2009)

15. Blum, E, Oettli, W: From optimization and variational inequalities to equilibrium problems. Math. Stud. 63, 123-145 (1994)

16. He, RH: Coincidence theorem and existence theorems of solutions for a system of Ky Fan type minimax inequalities in FC-spaces. Adv. Fixed Point Theory 2, 47-57 (2012)

17. Zegeye, $\mathrm{H}$ : A hybrid iteration scheme for equilibrium problems, variational inequality problems and common fixed point problems in Banach spaces. Nonlinear Anal. 72, 2136-2146 (2010)

18. Ye, J, Huang, J: Strong convergence theorems for fixed point problems and generalized equilibrium problems of three relatively quasi-nonexpansive mappings in Banach spaces. J. Math. Comput. Sci. 1, 1-18 (2011)

19. Qin, X, Cho, SY, Kang, SM: Iterative algorithms for variational inequality and equilibrium problems with applications. J. Glob. Optim. 48, 423-445 (2010)

20. Gu, F, Lu, J: Projection methods of iterative solutions in Hilbert spaces. Fixed Point Theory Appl. 2012, Article ID 162 (2012)

21. Kim, JK: Hybrid projection algorithms for generalized equilibrium problems and strictly pseudocontractive mappings. J. Inequal. Appl. 2010, Article ID 312602 (2010)

22. Kim, JK: Strong convergence theorems by hybrid projection methods for equilibrium problems and fixed point problems of the asymptotically quasi- $\boldsymbol{\phi}$-nonexpansive mappings. Fixed Point Theory Appl. 2011, Article ID 10 (2011)

23. Kim, JK, Anh, PN, Nam, YM: Strong convergence of an extended extragradient method for equilibrium problems and fixed point problems. J. Korean Math. Sci. 49, 187-200 (2012)

24. Yang, S, Li, W: Iterative solutions of a system of equilibrium problems in Hilbert spaces. Adv. Fixed Point Theory 1 , $15-26(2011)$

25. Qin, X, Shang, M, Su, Y: Strong convergence of a general iterative algorithm for equilibrium problems and variational inequality problems. Math. Comput. Model. 48, 1033-1046 (2008)

26. Luo, H, Wang, Y: Iterative approximation for the common solutions of a infinite variational inequality system for inverse-strongly accretive mappings. J. Math. Comput. Sci. 2, 1660-1670 (2012)

27. Cho, SY, Kang, SM: Approximation of common solutions of variational inequalities via strict pseudocontractions. Acta Math. Sci. 32, 1607-1618 (2012) 
28. Kang, SM, Cho, SY, Liu, Z: Convergence of iterative sequences for generalized equilibrium problems involving inverse-strongly monotone mappings. J. Inequal. Appl. 2010, Article ID 827082 (2010)

29. Cioranescu, I: Geometry of Banach Spaces, Duality Mappings and Nonlinear Problems. Kluwer Academic, Dordrecht (1990)

30. Alber, Yl: Metric and generalized projection operators in Banach spaces: properties and applications. In: Kartsatos, AG (ed.) Theory and Applications of Nonlinear Operators of Accretive and Monotone Type. Dekker, New York (1996)

31. Takahashi, W, Zembayashi, K: Strong and weak convergence theorems for equilibrium problems and relatively nonexpansive mappings in Banach spaces. Nonlinear Anal. 70, 45-57 (2009)

doi:10.1186/1029-242X-2013-387

Cite this article as: Zhao: Approximation of solutions to an equilibrium problem in a nonuniformly smooth Banach space. Journal of Inequalities and Applications 2013 2013:387.

Submit your manuscript to a SpringerOpen ${ }^{\circ}$ journal and benefit from:

- Convenient online submission

- Rigorous peer review

- Immediate publication on acceptance

- Open access: articles freely available online

- High visibility within the field

- Retaining the copyright to your article

Submit your next manuscript at $>$ springeropen.com 